\title{
Treating psoriasis with adalimumab
}

\author{
Eihab A Alwawi' \\ Stephanie L Mehlis' \\ Kenneth B Gordon ${ }^{1,2}$ \\ 'Division of Dermatology and \\ Department of Medicine, Evanston \\ Northwestern Healthcare, Skokie, IL, \\ USA; ${ }^{2}$ Department of Dermatology, \\ Northwestern University, Feinberg \\ School of Medicine, Chicago, IL, USA
}

\begin{abstract}
Psoriasis is a common, chronic, inflammatory skin disease that can have a significant impact on the quality of life of those who are afflicted. Recent advances in the understanding of the pathophysiology of psoriasis have led to the development of new, genetically engineered, targeted therapies for this disease. Among the most successful strategies for treatment has been the use of biologic immunotherapies targeting tumor necrosis factor alpha (TNF). Recent research has evaluated the efficacy and safety of a new anti-TNF agent, adalimumab. Adalimumab is a human monoclonal antibody that is approved by the US Food and Drug Administration (FDA) and the European Medicines Agency (EMEA) for the treatment of rheumatoid arthritis and psoriatic arthritis. Recently released data from large, randomized clinical trials suggests that adalimumab has significant efficacy for the treatment of chronic plaque psoriasis and is well tolerated. Thus, adalimumab seems to be a promising therapeutic approach for patients who suffer from moderate to severe plaque psoriasis.
\end{abstract}

Keywords: psoriasis, adalimumab, tumor necrosis factor alpha

Psoriasis is a chronic, inflammatory disease that affects approximately $2 \%-3 \%$ of the world's population. To date, this immune-mediated disease is incurable, and most commonly manifests itself as plaque-type psoriasis, which is characterized by the presence of red, thick, scaly lesions. It is an emotionally and physically debilitating disease that has a significantly negative impact on an affected person's quality of life (Krueger et al 2001). In the effort to improve treatment for patients who suffer from this disease, research has led to the discovery of several new therapies that directly target against the immune response that drives psoriasis. One specific protein that has proven as an effective target for therapy is tumor necrosis factor-alpha (TNF- $\alpha$ ). This article will provide a brief overview of current available biologic agents that target TNF- $\alpha$ in treatment of psoriasis, with particular emphasis on the human monoclonal antibody, adalimumab.

\section{Psoriasis and immunity}

Psoriasis is an example of an immune mediated disease. The interaction of multiple immune cell types including T-cells, macrophages, and dendritic cells induces abnormally rapid keratinocytic proliferation and incomplete maturation. These abnormalities result in thickening and scaling of the skin, the primary hallmarks of psoriasis.

One of the central immunological mediators in psoriasis is the cytokine TNF- $\alpha$. It is one of the major naturally occurring cytokines in the skin, and is involved in several normal and abnormal inflammatory immune responses, and is found in elevated levels in the skin of psoriatic patients (Rau 2002). TNF- $\alpha$ directly effects the pathogenesis of psoriasis, and demonstrates this by inducing the synthesis of adhesion molecules on endothelial cells and keratinocytes. This process thereby influences cellular infiltration in the skin, and has a direct effect on the abnormal keratinoctye proliferation and maturation seen in psoriatic lesions (Gottlieb 2003). 

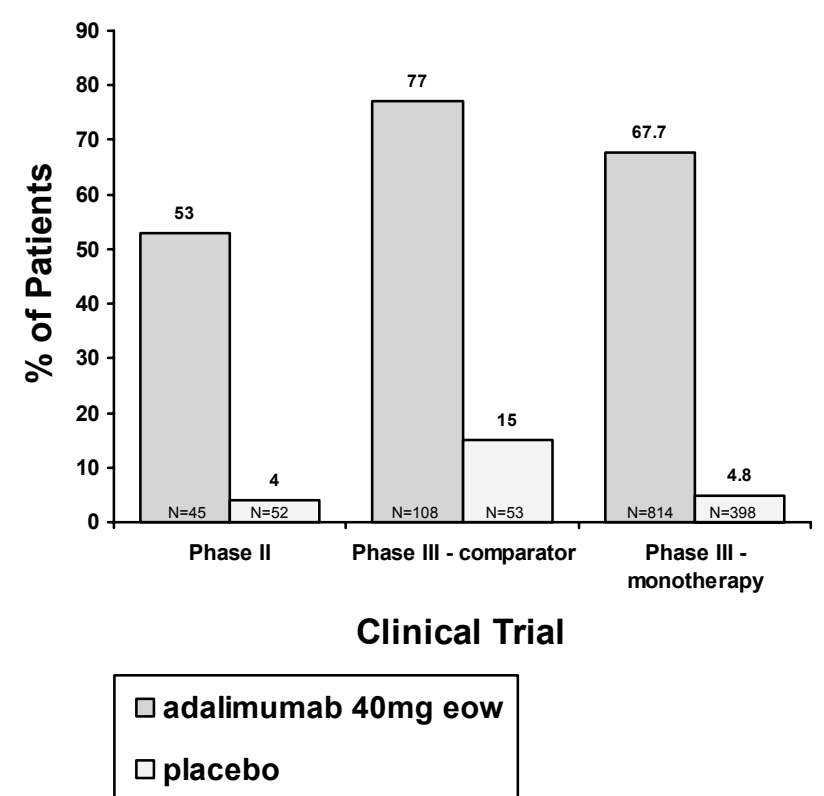

Figure I Percentage of patients achieving 75\% improvement in Psoriasis Area and Severity Index response at week 12 in the phase II and phase III trials. Because the phase II trial did not have week 16 data (the primary endpoint for the phase III trials), we compared the week I2 PASI 75 for all three clinical trials.

\section{Current methods of treatment: biologic agents}

Traditional treatments for moderate to severe psoriatic disease include phototherapy, systemic retinoids, methotrexate, and cyclosporine. However, as our understanding of the immunopathogenesis of psoriasis evolves, new directions in treatment of this disease have shifted to include newer biological agents that target specific immune cells and molecules, such as those responsible for the proliferation of keratinocytic changes seen in plaque psoriasis. Biological agents are proteins derived from recombinant DNA technology, hybridomas, blood, and whole human cells. In psoriasis, these agents are designed to specifically interfere with inflammatory cell activation. Some of these drugs, particularly those that target TNF- $\alpha$, are also effective in treating psoriatic arthritis (PsA) (Winterfield et al 2005).

There are several treatment methods available to block the induction or maintenance of $\mathrm{T}$ cell activation in psoriatic disease. One method that has proven successful involves inactivation of secreted effector cytokines. TNF- $\alpha$ appears to be a critical cytokine for many of the clinical features of psoriasis, including keratinocyte hyperproliferation, endothelial cell regulation, and recruitment/effector function of memory T cells (Winterfield et al 2005). Several anti-TNF drugs, such as etanercept, infliximab, and adalimumab, have been used successfully to treat psoriasis and PsA.

\section{Anti-TNF therapies}

Etanercept was the first drug approved by the US Food and Drug Administration (FDA) for the treatment of cutaneous psoriasis, and is administered twice weekly by subcutaneous injection. It is a fusion protein composed of human TNF type II receptor (TNF-Rp75) and human IgG1 Fc Region, which binds to soluble TNF- $\alpha$ (Papp 2006). In clinical trials, etanercept administered at a dose of $25 \mathrm{mg}$ subcutaneously twice weekly demonstrated statistically significant Psoriasis Area and Severity Index (PASI) 75 results (defined as a 75\% improvement from baseline) of $30 \%-34 \%$ at 12 weeks and $44 \%-56 \%$ at 24 weeks (Gottlieb et al 2003; Leonardi et al 2003). Greater efficacy was achieved using doses of $50 \mathrm{mg}$ subcutaneously twice weekly, resulting in PASI 75 responses of $49 \%$ at 12 weeks and $59 \%$ at 24 weeks (Moreland et al 2002). As with other anti-TNF drugs, risk of infection is a concern when using etanercept. In controlled trials, the rates of infections were not different from those in patients treated with placebo or methotrexate (Moreland et al 2002; Mease et al 2000; Gaylor and Duvic 2004). However, it should be noted that post-marketing safety surveillance has included reports of serious and, rarely, fatal infections in patients treated with anti-TNF agents (Winterfield et al 2005).

Infliximab, an FDA-approved drug for the treatment of severe psoriasis, was the first TNF blocker studied for the treatment of psoriasis. It is a chimeric monoclonal antibody that binds soluble and membrane-bound TNF$\alpha$ that is administered by intravenous infusion. Phase II trials studying infliximab demonstrated the efficacy of this medication for the treatment of psoriasis (Chaudhari et al 2001; Gottlieb et al 2004). A phase III, international, multi-center, randomized, placebo-controlled trial of adult patients with plaque psoriasis was performed to validate the results of the phase II program (Reich et al 2005). The study demonstrated that nearly $80 \%$ of subjects randomized to the infliximab arm achieved PASI 75 through 24 weeks of therapy (Moreland et al 2002). Since infliximab is a chimeric antibody, there is the potential for development of neutralizing antibodies (Winterfield et al 2004). In the phase III trial, infection rates were comparable between the treated and placebo groups and post marketing data from infliximab-treated patients with RA, Crohn's disease, or other indications for which infliximab is approved suggest a potential increased risk for events such as opportunistic infections (eg, tuberculosis), lymphoma, or congestive heart failure (Reich et al 2005). 


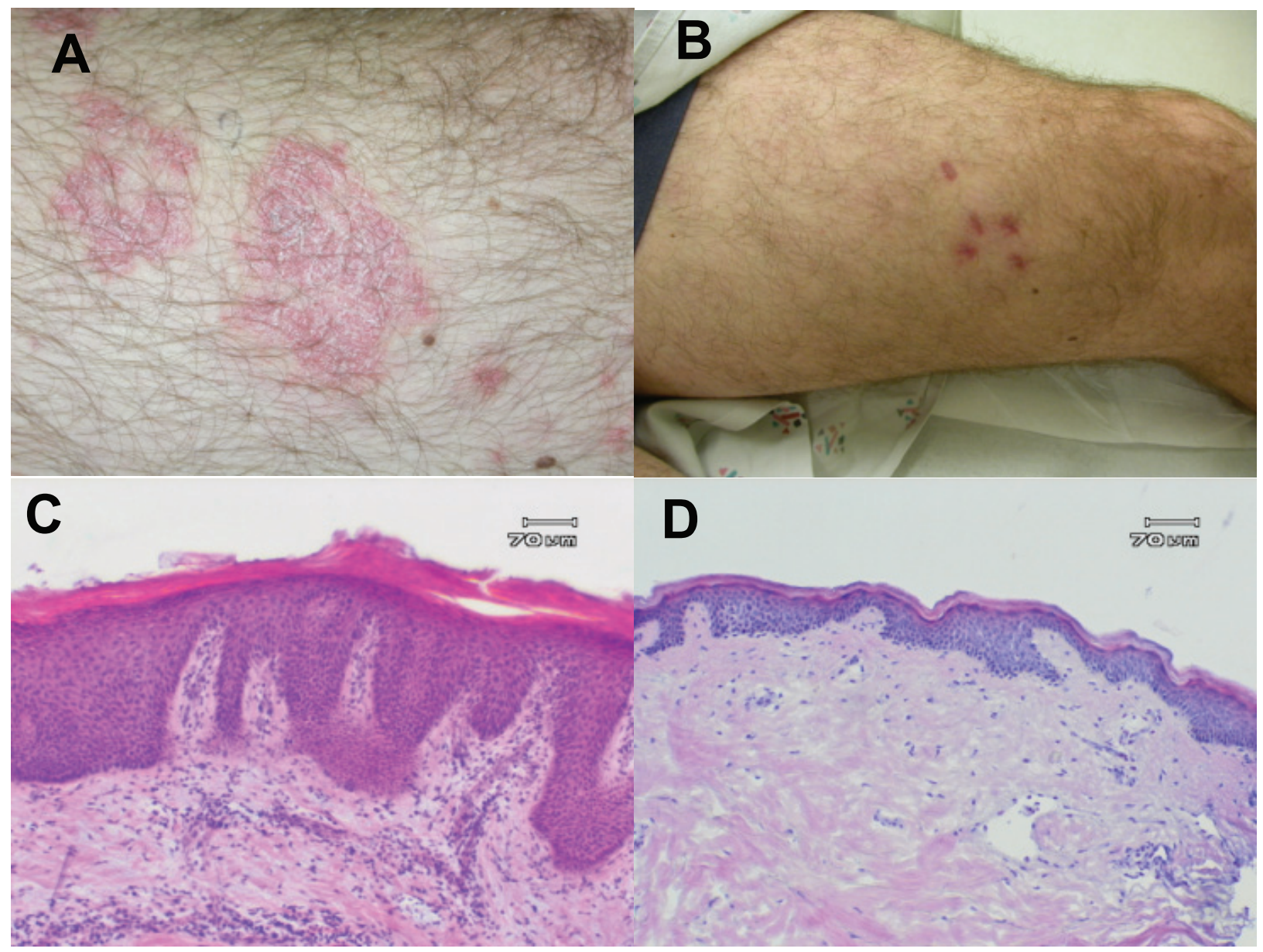

Figure 2 Clinical and histological response of subject in phase II study of adalimumab after 12 weeks. Clinical photos target lesion at day 0 (A) and day 84 (B) and representative histological specimens at day 0 (C) and day 84 (D).

\section{Role of adalimumab in treatment of psoriasis}

Adalimumab is currently FDA and European Medicines Agency (EMEA) approved for treatment of PsA and rheumatoid arthritis, for which it is administered every other week by subcutaneous injection. It is a recombinant, fully human Immunoglobulin G1 monoclonal antibody that binds with high affinity and specificity to TNF- $\alpha$ (Calabrese 2003). It consists of 1330 amino acids and has a molecular weight of approximately $148 \mathrm{kDa}$ (Krueger et al 2000). Once it is given, the drug reaches it peak level at 51/2 days, with a half-life of approximately 14 days (den Broeder et al 2002). The majority of responses attributed to TNF are regulated by the p55 cell surface TNF receptors. Upon binding to TNF- $\alpha$, adalimumab blocks its interaction with the p55 and p 75 cell surface TNF receptors. Adalimumab works by altering TNF-induced or regulated biological responses, such as changes in the levels of adhesion molecules responsible for leukocyte migration (Abbott Laboratories 2004). This process of neutralization of TNF- $\alpha$ by a specific monoclonal antibody should improve both skin and joint manifestations of psoriasis.

\section{Efficacy of adalimumab}

The efficacy of adalimumab has been studied in phase II and phase III clinical trials for the treatment of moderate to severe plaque-type psoriasis. The data are summarized in Figure 1. The first data were provided by the phase II, double-blind, placebo-controlled, randomized trial evaluating 147 subjects with psoriatic involvement of at least $5 \%$ of their body surface area (BSA) and a minimum PASI score of at least 10 (Gordon et al 1998). This dose-finding trial evaluated adalimumab as monotherapy without the addition of other systemic therapies, topical medication, or light therapy. The primary endpoint of the study was the percentage of subjects achieving at least a PASI 75 score at week 12. After week 12, subjects were followed and provided treatment for an additional 48 weeks through week 60. During the first 12 weeks, patients were randomized into one of three groups: 1) Placebo, 2) Low dose group 
$-80 \mathrm{mg}$ loading dose for 1 week, followed by $40 \mathrm{mg}$ every other week (eow), or 3) High dose group - $80 \mathrm{mg}$ loading dose for 2 weeks, followed by $40 \mathrm{mg}$ every week (ew). After week 12 , subjects in the placebo arm were crossed over to receive the eow regimen, while all other subjects continued on their prior dosing schedule. At the week 24 endpoint, subjects on the eow arm with disease recurrence up to $50 \%$ of their initial PASI (PASI 50) were permitted to dose escalate to $40 \mathrm{mg}$ weekly. Data were analyzed as a non-responder imputation (NRI) analysis, with any patient requiring dose escalation considered a treatment failure (Gordon et al 1998).

The results of this phase II study demonstrated the efficacy of adalimumab in the treatment of moderate to severe plaquetype psoriasis. In this trial, the primary endpoint of PASI 75 or better was achieved in $53 \%$ and $80 \%$ of the patients receiving adalimumab eow or every week, respectively, as compared to $4 \%$ of the patients receiving placebo. At week $60,56 \%$ of the eow group achieved PASI 75, as compared to $64 \%$ for the weekly group (Gordon et al 1998). Data of particular interest included a PASI 100 (total clearance of psoriasis) at week 60 in $16 \%$ of subjects in the low dose group (eow) and $26 \%$ of subjects in the high dose group (ew) (Gordon et al 1998). Additionally, clinically and statistically significant improvements in the Dermatology Life Quality Index (DLQI) were reported at week 60 (Shikiar et al 2006). Clinical and histological responses of a typical patient are shown in Figure 2.

Two phase III trials were devised to provide a greater body of data to validate the successful results of the initial phase II study. The first was a 16-week study performed in Europe and Canada, comparing the efficacy of adalimumab to methotrexate and placebo. The second study, conducted in North America, compared adalimumab monotherapy to placebo for longer term use (Menter et al 2007).

The comparator phase III trial was the first study to directly compare the clinical efficacy, safety, and tolerability of any biologic to a traditional systemic agent in the treatment of moderate to severe chronic plaque psoriasis (Saurat et al 2006). In this study, 271 subjects were randomized in a 2:2:1 ratio into one of three arms: 1) $80 \mathrm{mg}$ loading dose for 1 week, followed by $40 \mathrm{mg}$ every other week (eow) for the next 15 weeks, 2) methotrexate under a published regimen (Roenigk et al 1998), or 3) placebo. The main inclusion for participation included a clinical diagnosis of plaque- type psoriasis for at least 12 months, an affected BSA of at least $10 \%$, and a PASI score of 10 or greater. The primary endpoint of the trial was the percentage of subjects achieving a PASI 75 response rate at week 16 (Saurat et al 2006).
The results of the comparator study established that adalimumab provided significantly greater efficacy in the treatment of moderate to severe plaque- type psoriasis versus metho-trexate and versus placebo. The primary endpoint (PASI 75) was demonstrated in $80 \%$ of subjects in the adalimumab group, as compared to $36 \%$ in the methotrexate group, and 19\% in the placebo group. A Physician Global Assessment (PGA) score of "clear" or "minimal" was achieved in $73 \%$ of the adalimumab treated patients versus $30 \%$ in the methotrexate group and $11 \%$ in placebo (Saurat et al 2006).

While this trial successfully established the relative efficacy of adalimumab in comparison to methotrexate in the treatment of plaque- type psoriasis, there are some difficulties that should be mentioned. The achieved placebo control rate of PASI 75 was abnormally high at 19\%. However, an analysis of the treatment effect, a measure that subtracts for the placebo rate from the overall response, is $62 \%$, comparable to other trials with adalimumab (Menter et al 2007). Furthermore, although this study used a published protocol for methotrexate dosing, it has been argued that the methotrexate response would be higher with a more aggressive treatment regimen. However, it is important to acknowledge that a more aggressive approach may also result in greater rate of hepatic abnormalities (Heydendael et al 2003). Since the data analysis of this protocol was a non-responder imputation (NRI) analysis, it is not certain that a higher methotrexate dose would give a greater response rate. Therefore, it is valid to conclude that adalimumab showed significant efficacy advantages over methotrexate in this trial.

The North American phase III trial examined the short and long term efficacy of adalimumab as monotherapy versus placebo. This 52-week, randomized, double-blind, placebocontrolled, multicenter, phase III study evaluated 1,212 patients with moderate to severe chronic plaque psoriasis, defined a PASI $\geq 12$ and BSA $\geq 10 \%$ (Menter et al 2007). There were two independent primary endpoints in this trial. The first primary endpoint was the proportion of patients that achieved PASI $\geq 75$ at week 16 . The second primary endpoint was the percentage of patients losing an adequate response (defined as PASI $<50$ or a 6 point increase in PASI score) between weeks 33 and 52 (Menter et al 2007). The study was divided into three treatment periods (A, B, and C), and PASI 50/75/90/100 and PGA results were evaluated using non-responder imputation (NRI) analysis (Menter et al 2007). In the double-blind, placebo-controlled period A, patients were randomized $2: 1$ to receive $80 \mathrm{mg}$ of adalimumab at baseline (week 0 ) followed by $40 \mathrm{mg}$ eow, or placebo through week 15. At week 16, patients with PASI $>75$ entered period 
$\mathrm{B}$, at which point they would receive adalimumab $40 \mathrm{mg}$ eow from weeks 17 to 33. Patients who did not achieve PASI 75 at week 16 were enrolled into the open label extension (OLE) study. At week 33, patients who achieved PASI $\geq 75$ continued onto the double-blind, placebo-controlled period C and are re-randomized, 1:1 to receive adalimumab or placebo (Menter et al 2007). Loss of adequate response in this group is compared between weeks 33 and 52. Partial responders (PASI $50<75$ ) at week 33 were enrolled into the OLE study, and patients who were non-responders (PASI <50) at week 33 were discontinued from the study (Menter et al 2007). Patients originally randomized in period A to receive placebo and achieved PASI $\geq 75$ at week 16 , continued to receive adalimumab therapy in period $\mathrm{C}$ if they achieved PASI $\geq 75$ in period $\mathrm{B}$.

The first primary endpoint measured after 16 weeks of therapy, patients receiving adalimumab responded to their treatment at significantly higher rates than their placebo counterparts. Results from short-term therapy demonstrated that approximately $71 \%$ of patients achieved at least a $75 \%$ improvement of their psoriasis. Of interest, the mean percentage PASI improvement as compared to baseline was maintained at roughly $90 \%$, and nearly $23 \%$ of patients pooled from period $\mathrm{B}$ and the OLE study achieved $100 \%$ clearance of their psoriasis through 24 weeks of therapy (Menter et al 2007). In the second primary endpoint measured at week 52 , period C, $5 \%$ of patients receiving adalimumab experienced a loss of adequate response, significantly lower than the $28 \%$ of patients who were randomized to receive placebo during the same period.

The results of the phase III adalimumab monotherapy trial effectively demonstrates that treatment with adalimumab $40 \mathrm{mg}$ eow is highly efficacious for patients with moderate to severe plaque-type psoriasis. The study confirms that adalimumab's efficacy is sustainable as evidenced in the open label extension study, and discontinuation of adalimumab therapy is associated with loss of adequate response (Menter et al 2007).

Adalimumab may also be effective in patients who are refractory to other systemic agents. A small trial of 9 psoriatic patients who failed previous anti-TNF therapies found that adalimumab was an effective treatment for their psoriasis with $62.5 \%$ reaching a PASI 75 in 20 weeks (Pitarch et al 2007).

\section{Safety and tolerability of adalimumab}

Adalimumab was well tolerated in both phase II and III studies. In the phase II trial, the most reported adverse event was pain with injection, especially in the high dose group. Other adverse events were similar to placebo, with headache, nausea, elevated triglycerides, cough, sinus congestion, and fatigue most common. Two cancers were noted, however, review of patient histories determined that both cancers were likely present upon entry into the study (Gordon et al 1998). There were an increased number of serious adverse events (SAEs) in the highest dosing group in the phase II trial. However, this increase in SAEs was not observed in either the initial phase III trial, or the long-term phase III trial (Menter et al 2007). In the phase III comparator study, there were no significant differences in the incidences of adverse events reported for adalimumab-treated versus methotrexatetreated and placebo-treated patients, and the most frequent adverse events reported were nasopharyngitis and headache (Saurat et al 2006).

The safety profile observed in the phase III adalimumab monotherapy trial is consistent with earlier studies. In initial 16-week double-blind, placebo-controlled portion of the trial, period $\mathrm{A}$, the percentage of patients and rates of AEs and infectious AEs were greater in the adalimumab group as compared to the placebo group; however, less than $1 \%$ of the infectious AEs were considered serious (Menter et al 2007). The percentage of SAEs and serious infectious AEs in the adalimumab-treated group during period A was comparable to the rates experienced by the placebo group. During the first 16 weeks, a greater percentage of patients withdrawing study participation occurred in the placebo-arm experiencing AEs than in the adalimumab-treated group (Menter et al 2007). Additionally, the 52-week safety profile confirmed that the rates of SAEs, serious infectious AEs, and malignancies among adalimumab-treated patients were low and comparable to those patients in the placebo-arm in period A (Menter et al 2007). The most common side effects experienced by patients in the adalimumab treatment arm were upper respiratory infections (7.2\%), nasopharyngitis $(5.3 \%)$, headache $(4.9 \%)$, and injection site reactions $(3.1 \%)$ (Menter et al 2007).

To achieve a greater understanding of the safety and tolerability of adalimumab, it is helpful to review the data from clinical studies and post-marketing observations of the drug in the treatment of rheumatoid arthritis (RA). It is important to point out, however, that the RA patient population is typically quite different than that of psoriasis, as the RA population tends to be older and on other immunosuppressive agents, including methotrexate and systemic corticosteroids. With this in mind, there is still much that can be learned from the RA trials regarding the safety of adalimumab. 
Adalimumab's mechanism of action may suppress the human body's defenses against infections and malignancies, and therefore these risks are the greatest concern when using this type of medication. In randomized, placebo-controlled RA trials, the risk of serious infection is comparable, or slightly increased between groups treated with adalimumab and those on placebo when controlled for exposure duration (Furst et al 2003; Schiff et al 2006). Furthermore, the rate of serious infections seems to be most dependent upon the age of the patient and on the concominant use of systemic corticosteroids and/or methotrexate (Kent et al 2006; Schiff et al 2006). In another trial, the rates of infection were higher in methotrexate monotherapy as compared to monotherapy adalimumab, but the rates of infection were even greater when the two medications were combined (Breedveld et al 2006). These results indicate that risk of infection with adalimumab may be lower in younger psoriasis patients who are being treated with monotherapy (Winterfield et al 2005).

The possibility of reactivation of latent tuberculosis $(\mathrm{Tb})$ is an area of concern with the arrival of anti-TNF- $\alpha$ biologic therapies. In clinical trials with adalimumab, there were thirteen reported cases of $\mathrm{Tb}$ (Abbott Laboratories 2004). While cases of $\mathrm{Tb}$ were reported among all doses, the incidence was greatest at doses of adalimumab that were higher than the recommended dose (Abbott Laboratories 2004). Appropriate screening for $\mathrm{Tb}$ is important, as it can reduce the incidence of reactivation of $\mathrm{Tb}$ by $82 \%$ (Perez et al 2006).

Other than infection, cancer was the greatest concern of safety raised in the RA trials using adalimumab. Meta-analysis suggested that the incidence of solid tumors when evaluated without regard to critical values like duration of study period, was higher in adalimumab treated patients. However, when the data are normalized for length of follow-up, the data suggest that there is no noticeable increase in risk of cancer for RA patients on adalimumab as compared to what is observed in the general population (Schiff et al 2006). Similarly, the rate of lymphoma is what would be expected in RA patients being treated with adalimumab, although this rate is higher than the general population (Schiff et al 2006). Other potential side effects of adalimumab use in the RA population include demyelinating disease, worsening of congestive heart failure, and clinically apparent autoimmunity (Abbott Laboratories 2004).

One of the largest studies looking at the tolerability, effectiveness, and safety of adalimumab was a multi-center, open label clinical study with 6610 rheumatoid arthritis patients treated with adalimumab alone or in combination with standard disease-modifying antirheumatic drugs (DMARDs). The most notable serious adverse events observed in patients receiving adalimumab monotherapy were serious infections (1.7\%).
Table I Adverse events in adalimumab psoriasis trials

Common adverse events in phase III psoriasis trial of adalimumab (Menter et al 2007)

\begin{tabular}{ll}
\hline Adverse event & $\%$ reported \\
Upper respiratory infection & $7.2 \%$ \\
Nasopharyngitis & $5.3 \%$ \\
Headache & $4.9 \%$ \\
Injection site reactions & $3.1 \%$ \\
\hline
\end{tabular}

Serious adverse events in RA open label adalimumab trial (Burmester et al 2007)

\begin{tabular}{ll}
\hline Adverse event & $\%$ reported \\
Serious infections & $1.7 \%$ \\
Malignancies $^{\mathrm{a}}$ & $0.65 \%$ \\
Congestive heart failure $^{\mathrm{a}}$ & $0.3 \%$ \\
Demyelinating disease $^{\mathrm{a}}$ & $0.06 \%$ \\
Systemic lupus erythematosus & $0.03 \%$ \\
\hline
\end{tabular}

anclusive of adalimumab monotherapy and adalimumab plus disease-modifying antirheumatic drugs.

Rare SAEs such as malignancy $(0.65 \%)$, congestive heart failure $(0.3 \%)$, demyelinating disease $(0.06 \%)$, and systemic lupus erythematosus $(0.03 \%)$ were observed among the 6610 patients receiving adalimumab monotherapy and adalimumab plus DMARDs (including methotrexate). Table 1 summarizes the serious adverse events captured in this trial (Burmester et al 2007). Although these events are extremely rare, clinicians should use caution when using adalimumab therapy.

\section{Conclusion}

Psoriasis is a chronic and debilitating inflammatory skin disease that affects a significant proportion of the population. The advent of biologic agents, and specifically anti-TNF- $\alpha$ therapy, has provided clinicians with promising new treatment options for their patients' moderate to severe psoriatic disease. In clinical trials, adalimumab has demonstrated excellent efficacy in the treatment of more severe cases of psoriasis. Additionally, research subjects tolerated adalimumab quite well, and reported significant improvement in their quality of life. While more clinical data are needed to fully understand the risk associated with adalimumab use in psoriasis, current information demonstrates that this medication is an appropriate option for treatment of moderate to severe psoriasis.

\section{References}

Abbott Laboratories. 2004. Abbott Park IL. Adalimumab (Humira) package insert.

Breedveld FC, Weisman MH, Kavanaugh AF et al. 2006. The PREMIER study: A multicenter, randomized, double-blind clinical trial of combination therapy with adalimumab plus methotrexate versus methotrexate alone or adalimumab alone in patients with early, aggressive rheumatoid arthritis who had not had previous methotrexate treatment. Arthritis Rheum, 54:26-37. 
Burmester GR, Mariette X, Montecucco C, et al. 2007. Adalimumab alone and in combination with disease-modifying antirheumatic drugs for the treatment of rheumatoid arthritis in clinical practice: the Research in Active Rheumatoid Arthritis (ReAct) trial. Ann Rheum Dis, 66:732-9.

Calabrese LH. 2003. Molecular differences in anticytokine therapies. Clin Exp Rheumatol, 21:241-8

Chaudhari U, Romano P, Mulcahy LD, et al. 2001. Efficacy and safety of infliximab monotherapy for plaque-type psoriasis: a randomized trial. Lancet, 357:1842-7.

Den Broeder AA, van de Putte LBA, Rau R, et al. 2002. A single dose, placebo controlled study of the fully human anti-tumor necrosis factor-alpha antibody adalimumab (D2E7) in patients with rheumatoid arthritis. J Rheumatol, 29:2288-98.

Furst DE, Schiff MH, Fleischmann RM, et al. 2003. Adalimumab, a fully human anti tumor necrosis factor-alpha monoclonal antibody, and concominant standard anti-rheumatic therapy for the treatment of rheumatoid arthritis: results of STAR (Safety Trial of Adalimumab in Rheumatoid Arthritis). J Rheumatol, 30:2563-71.

Gaylor ML, Duvic M. 2004. Generalized pustular psoriasis following withdrawal of efalizumab. J Drugs Dermatol, 3:77-9.

Gordon KB, Langley RG, Leonardi C, et al. 1998. Clinical response to adalimumab treatment in patients with moderate to severe psoriasis: double-blind, randomized controlled trial and open-label extension study. J Am Acad Dermatol, 38:478-85.

Gottlieb AB, Evans R, Li S, et al. 2004. Infliximab induction therapy for patients with severe plaque-type psoriasis: a randomized, double-blind, placebo-controlled trial. J Am Acad Dermatol, 51:534-42.

Gottlieb AB. 2003. Infliximab for psoriasis. J Am Acad Dermatol, 49: S112-17.

Gottlieb AB, Matheson RT, Lowe N, et al. 2003. A randomized trial of etanercept as monotherapy for psoriasis. Arch Dermatol, 139:1627-32; discussion, 1632

Heydendael VM, Spuls PI, Opmeer BC, et al. 2003. Methotrexate versus cyclosporine in moderate-to-severe chronic plaque psoriasis. $N$ Engl $J$ Med, 349:658-65.

Kent J, Pangan A, Szczerbak N, et al. 2006. Serious infections in patients with rheumatoid arthritis in adalimumab clinical trials [abstract]. 15th Congress of the European Academy of Dermatology and Venereology (EADV), Rhodes, P023.116.2006.

Kreuger G, Koo J, Lebwohl M, et al. 2001. The impact of psoriasis on quality of life: results of a 1998 National Psoriasis Foundation patient membership survey. Arch Dermatol, 137:280-4.

Krueger J, Gottlieb A, Miller B, et al. 2000. Anti-CD11a treatment for psoriasis concurrently increases circulating $\mathrm{T}$-cells and decreases plaque T-cells, consistent with inhibition of cutaneous T-cell trafficking. J Invest Dermatol, 115:333.
Leonardi CL, Powers JL, Matheson RT, et al. 2003. Etanercept Psoriasis Study Group. Etanercept as monotherapy in patients with psoriasis. N Engl J Med, 349:2014-22.

Mease PJ, Goffe BS, Metz J, et al. 2000. Etanercept in the treatment of psoriatic arthritis and psoriasis: a randomized trial. Lancet, 356:385-90.

Menter A, Tyring SK, Gordon K, et al. 2007. Adalimumab therapy for moderate to severe psoriasis: A randomized, controlled phase III trial. $J$ Am Acad Derm. In press.

Moreland LW, Schiff M, Cohen S, et al. 2002. Safety and efficacy of up to 5 years of etanercept (Enbrel) therapy in rheumatoid arthritis. Presented at the European League Against Rheumatism, 12-15 June, 2002.

Papp KA. 2006. The long-term efficacy and safety of new biological therapies for psoriasis. Arch Dermatol Res, 298:7-15.

Perez J, Kupper H, Okun M. 2006. Outcomes of screening for latent Tb in worldwide adalimumab clinical trials [abstract]. 15th Congress of the European Academy of Dermatology and Venereology (EADV), Rhodes, P035.100. 10-1-2006.

Pitarch G, Sanchez-Carazo JL, Mahiques L, et al. January 2007. Treatment of Psoriasis with Adalimumab. Clin Exp Dermatol, 32:18-22.

Rau R. 2002. Adalimumab (a fully human anti-tumor necrosis factor alpha monoclonal antibody) in the treatment of active rheumatoid arthritis: the initial results of five trials. Ann Rheum Dis, 61(suppl 2):ii70-73.

Reich K, Nestle FO, Papp K, et al. 2005. EXPRESS study investigators (2005) Infliximab induction and maintenance therapy for moderateto-severe psoriasis: a phase III, multicentre, double-blind trial. Lancet, 366:1367-74.

Roenigk HH Jr, Auerbach R, Maibach H, et al. 1998. Methotrexate in psoriasis: consensus conference. J Am Acad Dermatol, 38:478-85.

Saurat J, Stingl G, Dubertrel L, et al. 2006. CHAMPION Phase III Trial Results: Adalimumab Efficacy and Safety Compared with Methotrexate and Placebo in Patients with Moderate to Severe Psoriasis [abstract]. 15th Congress of the European Academy of Dermatology and Venereology (EADV), Rhodes, P035.165.2006.

Schiff MH, Burmester GR, Kent JD, et al. 2006. Safety analyses of adalimumab (HUMIRA) in global clinical trials and US postmarketing surveillance of patients with rheumatoid arthritis. Ann Rheum Dis, 65:889-94.

Shikiar R, Willian MK, Okun MM, et al. 2006. The validity and responsiveness of three quality of life measures in the assessment of psoriasis patients: results of a phase II study. Health Qual Life Outcomes, $4: 71$.

Winterfield LS, Menter A, Gordon K, et al. 2005. Psoriasis treatment: current and emerging directed therapies. Ann Rheum Dis, 64(Suppl 2): ii87-90. 
